\title{
13 Ethische Herausforderungen bei psychisch bedingten Notfallsituationen
}

Frank Lasogga

\subsection{Was für Notfallpatienten und Situationen gibt es?}

Zunächst einmal ergibt sich die Frage: Was sind psychisch bedingte Notfallsituationen? Bereits diese Frage ist nicht einfach zu beantworten. Bei einer psychisch bedingten Notfallsituation werden viele sicherlich sofort daran denken, dass jemand eine psychische Störung wie eine Depression, Schizophrenie oder eine Suchterkrankung hat und dass sich im Umgang mit diesen Personen ethische Herausforderungen ergeben.

Einige der relevanten psychischen Störungen sind im Folgenden kurz skizziert. Zur Beschreibung und Diagnose psychischer Störungen wird das Diagnostische und Statische Manual Psychischer Störungen DSM 5 (Falkai u. Wittchen 2015) herangezogen. Das International Statistical Classification of Diseases and Related Health Problems ICD 1o (Dilling et al. 2011), das ebenfalls eine allerdings kürzere Beschreibung enthält, und das DSM haben sich in den letzten Jahren immer stärker angeglichen. Deshalb wird hier nur das DSM herangezogen, zumal dies auch neueren Datums ist.

\subsubsection{Depressive Notfallpatienten}

Im DSM 5 wird eine ganze Reihe von depressiven Störungen aufgeführt. Den klassischen Prototyp dieser Störungsgruppe repräsentiert die Major Depression. Sie ist u.a. gekennzeichnet durch depressive Episoden. Diese müssen für die Diagnose zwei Wochen dauern, sind meistens aber länger und „durch klar abgrenzbare Veränderungen im Affekt, der Kognition und neurovegetativen Funktionen sowie Remission zwi- 
schen den Episoden charakterisiert" (Falkai u. Wittchen 2015: 209). In diesen Episoden treten u.a. eine depressive Verstimmung an fast allen Tagen für die meiste Zeit des Tages, deutlich vermindertes Interesse oder Freude an fast allen Aktivitäten etc. auf und die Symptome verursachen in klinisch bedeutsamer Weise Leiden oder Beeinträchtigungen. Ein Suizidrisiko ist während der gesamten Zeit vorhanden. Eine nicht geringe Anzahl von Personen, die einen Suizid begehen, ist an einer Depression erkrankt (gewesen). Aber nicht nur bei einem Suizidversuch kann es zu Problemen kommen, sondern auch, wenn beispielsweise interveniert werden muss, weil eine Mutter aufgrund der Depression ihr Kind vernachlässigt.

\subsubsection{Schizophrene Notfallpatienten}

Zum Schizophrenie-Spektrum gehören eine ganze Reihe von Störungen. Sie sind durch eines oder mehrere Hauptmerkmale gekennzeichnet wie: „Halluzinationen, Wahn, desorganisiertes Denken (desorganisierte Sprache), grob desorganisiertes Verhalten oder gestörte Motorik “ (Falkai u. Wittchen 2015: 117). Bei den von Schizophrenie Betroffenen, eine Form der Störungen aus dem Schizophrenie-Spektrum, begehen gemäß DSM 5 6\% Suizid, 20\% unternehmen einen Suizidversuch und viele haben ausgeprägte Suizidvorstellungen.

\subsubsection{Borderline-Störung}

Patienten mit einer Borderline-Störung weisen ein „tiefgreifendes Muster von Instabilität in zwischenmenschlichen Beziehungen, im Selbstbild und in den Affekten sowie deutliche Impulsivität" auf (Falkai u. Wittchen 2015: 909). Die meisten der an einer Borderline-Störung Erkrankten denken an Suizid, ca. 5-10\% begehen Suizid. Die Borderline-Störung tritt allerdings häufig zusammen mit anderen Persönlichkeitsstörungen auf, sodass diese Zahl mit Vorsicht zu genießen ist.

\subsubsection{Störung durch Alkoholkonsum}

Alkoholabhängigkeit, im DSM 5 definiert als Alkoholkonsumstörung, ist weit verbreitet. Für die BRD wird häufig die Zahl von ca. 1,5 Millionen Abhängigen angegeben. Um von einer Alkoholkonsumstörung zu sprechen, müssen im DSM 5 mindestens 2 von 11 Kriterien innerhalb eines Zeitraums von 12 Monaten erfüllt sein. Andere Störungen wie Schizophrenie oder bipolare Störungen (aus dem Formenkreis der Depression) sind häufig mit der Alkoholkonsumstörung verbunden. Alkoholabhängige sind verstärkt suizidgefährdet.

\section{Alkoholkonsumstörung gemäß DSM 5}

A. Alkoholkonsum führt in klinisch bedeutsamer Weise zu Beeinträchtigungen oder Leiden. Mindestens zwei der Kriterien müssen innerhalb von 12 Monaten erfüllt sein:

1. Alkohol häufig in größeren Mengen oder länger als beabsichtigt konsumiert

2. Anhaltender Wunsch oder erfolglose Versuche, Konsum zu verringern oder kontrollieren

3. Hoher Zeitaufwand für Beschaffung, Konsum oder Erholung von Wirkung 
4. Craving oder starkes Verlangen nach Konsum

5. Wiederholter Konsum, der zu Versagen bei Erfüllung wichtiger Verpflichtungen führt

6. Fortgesetzter Konsum trotz ständiger sozialer und zwischenmenschlicher Probleme, die durch Auswirkungen von Alkohol bedingt sind

7. Wichtige soziale, berufliche Aktivitäten aufgrund des Konsums aufgegeben oder eingeschränkt

8. Wiederholter Konsum in Situationen, in denen Konsum zur körperlichen Gefährdung führt

9. Fortgesetzter Konsum trotz körperlichem oder psychischem Problem aufgrund des Konsums

10. Toleranzentwicklung

a. Verlangen nach ausgeprägter Dosissteigerung für erwünschten Effekt

b. Deutlich verminderte Wirkung derselben Menge

11. Entzugssymptome

a. Charakteristisches Entzugssymptom in Bezug auf Alkohol

b. Konsum, um Entzugssymptome zu lindern oder zu vermeiden

\subsubsection{Aggression}

Personen können aggressiv gegenüber anderen sein, aber Aggressionen können sich auch gegen sich selbst richten. In beiden Fällen muss die Handlungsfreiheit eingeschränkt werden, auch wenn die Personen dies nicht wollen. Damit erhebt sich die Frage, ab welchem Punkt eine derartige Einschränkung des Einzelnen gerechtfertigt ist und wie weit diese gehen kann. Im DSM 5 ist keine Störung „Aggression“ vorhanden, wohl aber die „Antisoziale Persönlichkeitsstörung“, die sich u.a. durch Reizbarkeit und Aggressivität äußert.

\subsubsection{Suizid, Suizidversuch}

Die dargestellten und andere Störungen können zu einem Suizidversuch oder zu Suizid führen, sodass auch dieses Thema behandelt werden soll. Im wissenschaftlichen Bereich wird der Begriff Suizid statt anderer Begriffe verwandt. Dieser Begriff hat eine neutrale Konnotation. Begriffe wie „Freitod“ oder „Selbstmord“ haben eine positive oder negative Konnotation.

In der BRD unternehmen jährlich ca. 10.0oo Menschen einen Suizid. Die Zahl liegt vermutlich höher, da viele Suizide beispielsweise als Unfälle ausgegeben werden. Wesentlich höher - Schätzungen gehen davon aus, dass die Zahl 10-30-mal so hoch ist - liegt die Zahl an Suizidversuchen.

Ein Suizid bzw. Suizidversuch kann im Zusammenhang mit einer Störung begangen werden, aber auch andere Umstände sind möglich, beispielsweise der sogenannte Bilanzsuizid. Die Positionen zur Berechtigung eines Suizids gehen weit auseinander. Eine Position besagt, dass der Mensch das Recht auf einen Suizid hat. Die gegenteilige Position wird ebenso vertreten, nämlich dass der Mensch kein Recht auf Suizid hat. Beide Positionen sind bereits bei Philosophen in der Antike zu finden und ziehen 
sich seitdem durch die Philosophie. Eine weitere Position besagt, dass jeder Mensch psychisch gestört ist, der einen Suizidversuch unternimmt oder einen Suizid begeht.

\section{Das Recht auf Suizid}

Von den Vertretern dieser Position wird argumentiert, dass jeder Mensch ein Recht auf einen Suizid hat. Der Mensch könne infolge einer Bilanz frei-willig (also aus freiem Willen) Suizid begehen, und dieses Recht stehe ihm zu. Der Mensch habe das Recht auf Selbstbestimmung und Autonomie. Auch könne das Leben zu solch einer Last werden, dass jemand den Tod vorzieht. Wenn jemand sagt: „Ich will sterben“, hat eine andere Person demnach nicht das Recht zu sagen: „Du darfst das nicht. Du siehst das falsch. Ich sehe das richtig. Du musst am Leben bleiben. "Eine derartige Haltung, in der die Autonomie des Menschen betont wird, wird philosophisch begründet.

Eng damit hängen auch Überlegungen zur indirekten und passiven Sterbehilfe („Sterbenlassen“) zusammen. Bei der indirekten Sterbehilfe können medizinische Maßnahmen eingesetzt werden, die das Leiden vermindern, aber gleichzeitig das Leben durch den Einsatz dieser Maßnahmen verkürzen. Bei dem „Sterbenlassen“ (international wird dafür häufig der Begriff „passive Sterbehilfe“ verwendet) wird auf lebensverlängernde oder lebenserhaltende Maßnahmen verzichtet. So kann die künstliche Ernährung eingestellt oder ein Sauerstoffgerät abgeschaltet werden. Berücksichtigt wird dabei häufig der mutmaßliche Wille des Patienten.

\section{Kein Recht auf Suizid}

Auch die gegenteilige Haltung wird philosophisch und auch theologisch begründet. Demnach hat kein Mensch das Recht, Suizid zu begehen, sondern ist zum Leben verpflichtet. Begründet wird dies u.a. damit, dass allein Gott über das menschliche Leben verfügen darf. Außerdem verstoße Suizid gegen das Prinzip der Eigenliebe und Nächstenliebe.

\section{Suizid im Zusammenhang mit einer psychischen Erkrankung}

Suizid wird häufig von Personen begangen, die unter einer psychischen Erkrankung leiden. Insbesondere sind hier Störungen aus dem depressiven Formenkreis zu nennen, ferner Drogen- und Alkoholabhängigkeit sowie schizophrene Störungen. Dass diesen Personen Hilfe anzubieten ist, steht außer Frage. Allerdings ist auch hier zu fragen, inwieweit diese Menschen ein Recht auf Suizid haben. Möglicherweise würden diese Menschen auch nach einer Psychotherapie Suizid begehen. Hier spielt wieder der Gedanke, dass ein nicht psychisch Beeinträchtigter keinen Suizid begeht, eine zentrale Rolle.

Eine weitere Position besagt, dass Suizid nicht nur im Zusammenhang mit psychischen Erkrankungen auftritt, sondern dass jeder Mensch, der Suizid bzw. einen Suizidversuch begeht, psychisch gestört ist. Dabei wird davon ausgegangen, dass der Realitätsbezug verzerrt, der Suizid irrational und impulsiv und Ausdruck einer psychischen Störung sei (Fenner 2008: 93). Der dies feststellt, ist aber gesund, und er könne das entscheiden. Damit hat er nicht nur das Recht, den Menschen vom Suizid abzuhalten, sondern auch die Pflicht. Des Weiteren habe ein jeder Mensch die Pflicht zu leben. Damit muss man einen Menschen zum Leben zwingen, auch wenn er dies 
nicht will. Dieses Menschenbild ist normativ. Es wird davon ausgegangen, dass kein Mensch Suizid begehen würde, wenn er nicht psychisch gestört ist. Ein Suizid aufgrund einer freiwilligen klaren Entscheidung ist hierbei ausgeschlossen. Andere verneinen eine derartige Sichtweise allerdings vehement.

\section{Folgen eines Suizidversuchs}

Ein Suizidversuch kann diverse Folgen nach sich ziehen. Eine Folge können schwere gesundheitliche Schäden sein. Ein weiteres Problem können die Angehörigen sein. Die Angehörigen können sich einerseits Vorwürfe machen, dass die Person einen Suizidversuch unternommen hat. Andererseits können sie aber auch eine Mitschuld an dem Suizidversuch tragen, sehen dies aber nicht ein. Sie sind möglicherweise nicht angemessen mit dem Menschen umgegangen, oder ihnen ist es mehr oder minder gleichgültig, ob die Person einen Suizidversuch unternommen hat. Es erhebt sich dann die Frage, wie sie nach dem Suizidversuch mit dem Suizidalen umgehen und damit auch die Frage, wie mit den Angehörigen umgegangen werden soll. Schließlich zeigt ein Suizidversuch häufig nur eine kurzfristige Wirkung in der Richtung, dass Aufmerksamkeit erzielt wird, die aber bald wieder nachlässt. Manche Angehörige stellen auch nach kurzer Zeit ihre Bemühungen wieder ein.

Vor diesem Hintergrund erhebt sich die Frage, wie ein Rettungsteam mit Personen umgehen soll, die einen Suizidversuch unternommen, aber überlebt haben. Wenn eine realistische Chance besteht, dass keine starken physischen oder psychischen Beeinträchtigungen im Fall des Überlebens bestehen bleiben, ist nach Ansicht des Autors alles zu unternehmen, dass die Person überlebt. Aufgrund des Suizidversuchs kann sich die Lebenssituation ändern, sei es, dass die Person therapeutische Hilfe bekommt, die bewirkt, dass sie eine lebensbejahende Haltung einnimmt, sei es, dass Angehörige ihr Verhalten im Umgang mit der Person ändern, oder aufgrund einer anderen Änderung.

Es sind aber auch andere Situationen denkbar: Eine Person kann im Koma liegen, wenn das Rettungsteam vor Ort erscheint und die Person hat einen Abschiedsbrief hinterlassen, aus dem hervorgeht, dass sie nicht mehr leben will. In diesem Fall wird letztendlich das Verhalten des Rettungspersonals davon abhängig sein, ob die Beteiligten meinen, dass ein Mensch ein Recht auf einen Suizid hat oder nicht. In ersterem Fall wird es eher zugelassen werden, dass die Person stirbt, in letzterem Fall wird alles unternommen werden, dass die Person überlebt.

\subsubsection{Reanimation}

Eine weitere Problematik stellt die Frage der Reanimation (nicht nur bei Suizidalen) dar. Teilweise muss unter Zeitdruck und bei Informationsdefiziten entschieden werden, ob mit Reanimationsmaßnahmen begonnen werden soll und wann sie abgebrochen werden sollen. Die Entscheidung fällt nicht nur unter rein medizinischen Gesichtspunkten, sondern auch Variablen wie der Patient, die Situation und natürlich der Arzt sind von Bedeutung. Bei niedrigerem sozialem Status, mehrfachen Suizidversuchen, Demenz oder geistiger Retardierung wird seltener eine Reanimation eingeleitet. Hinsichtlich des Arztes sind die Berufserfahrung und die Gruppenzugehörigkeit relevant: Chirurgen brechen eine Reanimation früher ab als andere Gruppen (Hick et al. 2000). 


\subsubsection{Unterschiedliche Positionen}

Wie im Falle einer Reanimation oder eines Suizidversuchs vorgegangen werden soll, kann zu Konflikten im Rettungsteam führen. Beispielsweise kann es beim Auffinden einer älteren leblosen Person oder bei einer nach einem Suizidversuch sehr schwer verletzten Person bei den Mitgliedern eines Rettungsteams sehr unterschiedliche Vorstellungen über das Vorgehen geben. Einige vertreten die Auffassung, keine Reanimationsversuche zu unternehmen, während andere mit Reanimationsmaßnahmen beginnen möchten (Lasogga u. Karutz 2012).

Dies ist sicherlich kein günstiger Zeitpunkt, die Standpunkte lange auszudiskutieren. Faktisch sieht es so aus, dass die ranghöchste Person die Entscheidung fällt. Allerdings kann diese Person auch die eigene Meinung zur Diskussion stellen und sich überstimmen lassen. Eine Entscheidung sollte von den übrigen Mitgliedern des Rettungsteams in diesem Moment akzeptiert werden. Sie sollte aber im Nachhinein diskutiert werden, allerdings ohne dass dieser Person Vorwürfe gemacht werden. Letztendlich sollte die Diskussion dazu führen, dass Leitlinien festgelegt werden, wie in derartigen Situationen vorgegangen wird. Schließlich muss schnell eine Entscheidung gefällt werden und es kann beispielsweise kein Ethikkomittee herangezogen werden.

\subsubsection{Todesnachricht mitteilen}

Ein ethisches Problem kann sich bei der Mitteilung einer Todesnachricht ergeben. So kann eine Person, die augenscheinlich nicht überleben wird, explizit nach ihren Überlebenschancen fragen. Es steht die Entscheidung aus, ob ihr dies mitgeteilt werden soll. Für eine Mitteilung spricht, dass Menschen in den letzten Minuten ihres Lebens nicht belogen werden sollten. Dagegen spricht, dass es einigen Personen sehr schwerfallen könnte, mit dieser Information umzugehen, und sie leidend aus dem Leben scheiden. Als Kriterium könnte letzten Endes die Einschätzung des Patienten gesehen werden, ob dieser es verkraftet. Keine Rolle sollte bei der Entscheidung spielen, ob diese Mitteilung dem Helfer (Arzt, Rettungsdienstmitarbeiter, Notfallseelsorger etc.) schwerfällt. Natürlich ist eine derartige Haltung nicht einfach, aber ,it's part of the job“. Hilfreich ist es, sich mit dieser Problematik und eng damit verbunden auch mit der eigenen Person auseinanderzusetzen und entsprechende Fortbildungen zu besuchen oder die eigene Position beispielsweise in Supervisionsgruppen zu reflektieren.

Eine weitere Problematik kann auftreten, wenn jemand beispielsweise selbst schwer verletzt ist und nach dem Überleben von Angehörigen fragt. Auch hier erhebt sich die Frage, ob ihm wahrheitsgemäß mitgeteilt werden sollte, dass sie tot sind. In diesem Fall kann manchmal auf Zeit gespielt werden, beispielsweise in dem man sagt, dies kann momentan nicht mit Sicherheit gesagt werden, aber man werde sich darum kümmern. Die Mitteilung könnte dann erfolgen, wenn mehr Personal zur Verfügung steht, beispielsweise auch wenn ein Notfallseelsorger gerufen werden konnte (Lasogga 2011). 


\title{
13.2 Autonomie, Urteilsfähigkeit
}

Bei vielen Entscheidungen stellt sich auch die Frage der „Autonomie“ bzw. „Urteilsfähigkeit“. Beide Begriffe sind sehr komplex. Sie sind nicht eindeutig definiert und können wohl auch nicht eindeutig definiert werden. Einigkeit besteht allerdings darin, dass Personen aufgrund einer Störung wie Schizophrenie oder aufgrund von starkem Alkoholkonsum nicht oder nur eingeschränkt urteilsfähig sein können. Bei Extremfällen ist eine derartige Einschätzung unproblematisch, problematisch sind jedoch die Fälle, die nicht eindeutig sind. Weniger problematisch ist es, wenn ein Patient bereits in einer Patientenverfügung für diesen Fall Vorgaben gemacht hat. Ansonsten ist die vertretungsberechtigte Person zu informieren und deren Einverständnis einzuholen.

\subsection{Von Entscheidungen betroffene Personengruppen}

Von den Entscheidungen der Helfer können viele Personen betroffen sein. Die Meinung dieser Personen muss sich nicht decken, sie können andere Meinungen haben, wobei auch die Interessen unterschiedlich sein können.

\begin{abstract}
Ein berühmtes Beispiel für unterschiedliche Meinungen ist die Patientin Terri Schiavo (Wikipedia Zugriff am 11.5.2015). Terri Schiavo hatte eine durch Sauerstoffmangel bedingte schwere Gehirnschädigung erlitten. Die Eltern und der Ehemann beurteilten die Situation unterschiedlich und waren sich uneinig über die Fortsetzung lebenserhaltender Maßnahmen. Die Eltern meinten, eine Heilung oder eine signifikante Besserung sei möglich. Diese Ansicht wurde nur von wenigen Fachleuten geteilt, der Ehemann gehörte nicht dazu. Er verwies auch darauf, dass seine Ehefrau mehrmals den Wunsch geäußert hatte, in einem derartigen Fall nicht künstlich am Leben gehalten werden zu wollen. Die Auseinandersetzung wurde schließlich über Jahre hinweg vor Gericht ausgetragen und beschäftigte auch die Medien sehr stark. Die künstliche Ernährung wurde insgesamt 3-mal eingestellt. Vor Gericht hatten die Eltern 2-mal erreicht, dass die künstliche Ernährung wieder aufgenommen und die künstliche Magensonde eingeführt wurde. Insgesamt befassten sich 19 Richter mit dem Verfahren, ferner auch Politiker. Terri Schiavo lag 15 Jahre lang im Wachkoma. Nach dem Tod wurde die Leiche obduziert. Dabei stellte sich heraus, dass ihre Hirnschädigung so stark war, dass keine Behandlung eine Verbesserung bewirkt hätte. Dieser Fall führte nicht nur in den USA zu einer großen Diskussion über Sterbehilfe bzw. Sterbenlassen.
\end{abstract}

Wie ersichtlich, ist bei Angehörigen die Entscheidung nicht immer einheitlich oder eindeutig. Sie kann zwischen verschiedenen Angehörigen divergieren, aber auch intraindividuell kann eine Entscheidung schwerfallen. Auf der einen Seite möchte man nicht mit ansehen, wie eine Person leidet, wenn sie weiterlebt, aber man möchte auch nicht, dass sie nicht mehr lebt.

Angehörige können schließlich aus ganz banalen Gründen ein Interesse haben, dass jemand noch lebt oder nicht mehr lebt. Wenn die Person lebt, haben sie jemand, den sie versorgen können, und damit eine Aufgabe. In diesem Fall steht nicht das Wohl des Patienten im Vordergrund, sondern das eigene. Beide Wünsche, dass der Patient leben und dass er sterben soll, können aber beim Angehörigen auch gleichzeitig vorhanden sein. Die Gefühle und Entscheidungen eines Menschen sind nicht wie eine 
mathematische Lösung: Man muss nur lange genug über ein Problem nachdenken und dann hat man die Lösung. Sich widersprechende Gefühle können gleichzeitig vorhanden sein, und auch sich widersprechende Gedanken können gleichzeitig vorhanden sein.

Weitere betroffene Personengruppen können beispielsweise das Pflegepersonal sein. Sie haben möglicherweise eine Beziehung zu dem Patienten aufgebaut und sind auch emotional von der Angelegenheit betroffen. Das Pflegepersonal kann leiden, wenn der Patient weiterlebt, aber es kann auch leiden, wenn er stirbt. Hinzu kann auch der Druck von außen kommen, von Freunden, Bekannten, der Kirche oder auch der Gesellschaft. Da eine Diskussion oft ausgeklammert wird - wie überhaupt das Thema Tod und Sterben -, sind die Meinungen dazu oft nicht sehr reflektiert.

Bei derartigen Entscheidungen spielt natürlich auch eine Rolle, was ein Patient gewollt hätte. Darauf kann, wenn ein Patient nicht gefragt werden kann, allerdings nur geschlossen werden, und das Ergebnis kann unterschiedlich ausfallen. Eindeutiger sieht es aus, wenn ein Patient für einen derartigen Fall eine Patientenverfügung hinterlassen hat. Liegt diese nicht vor oder ist nicht bekannt, ist nach seinem mutmaßlichen Willen zu entscheiden.

\subsection{Aufarbeitung}

Entscheidungen in den oben skizzierten Situationen sind nicht einfach, zumindest wenn man reflektiert mit diesen Situationen umgeht. Häufig wird eine Unsicherheit bleiben, ob die Entscheidung richtig war. Deshalb ist es hilfreich, über diese Entscheidungen zu sprechen. Derartige Gespräche entlasten, Gedanken werden neu strukturiert, wenn über etwas gesprochen wird. Außerdem können Gedanken von Kollegen oder anderen Personen zu einer anderen Sichtweise führen, oder die eigene Sichtweise kann verstärkt werden.

Für Gespräche bieten sich mehrere Möglichkeiten an: das Gespräch mit Kollegen, mit der Familie oder Freunden. Derartige Gespräche werden häufig von Personen in helfenden Berufen genutzt. Darüber hinaus bietet sich eine Supervisionsgruppe an. $\mathrm{Zu}$ empfehlen ist, eine Supervision regelmäßig 2-3-mal im Jahr durchzuführen, außerdem, wenn akuter Bedarf besteht. In dieser Cruppe kann besprochen werden, wie mit Fällen umgegangen worden ist und auch wie man selbst psychologisch reagiert hat. Die Möglichkeit von Supervision sollte durch die Organisation bereitgestellt werden.

\subsection{Fazit}

Entscheidungen im Umgang mit psychisch bedingten Notfallsituationen bleiben immer schwer, einfache Antworten auf derartig komplexe Situationen kann es nicht geben. Auch philosophische Gedanken wie der Kategorische Imperativ von Kant: „Handle so, dass die Maxime deines Willens jederzeit zugleich als Prinzip einer allgemeinen Gesetzgebung gelten könnte“ bieten letztendlich keine Entscheidungshilfe. Sie stellen nur eine allgemeine Grundlage dar, die interpretiert werden muss. Es gibt letztendlich keine objektiv ableitbaren Kriterien. 
Für den Suizid gilt, dass ein dichotomes Denken, ein Mensch begehe entweder einen Bilanzsuizid oder weil er psychisch beeinträchtigt ist, zu kurz greift. Bei den meisten Menschen lässt sich dies nicht eindeutig sagen, und es kommen viele Faktoren zusammen.

Bei der Bewertung einer Handlung spielen auch die Zeit und die Kultur eine Rolle. Zurzeit ist in den Beneluxländern beispielsweise die aktive Sterbehilfe unter bestimmten Bedingungen erlaubt, in der BRD nicht. Das Sterbenlassen (passive Sterbehilfe) ist derzeit gemäß geltender Rechtsprechung in den meisten europäischen Ländern erlaubt. Die Beihilfe zum Suizid (assistierter Suizid) ist in den europäischen Ländern und den Staaten der USA teilweise erlaubt, teilweise verboten.

Vergessen werden sollte dabei auch nicht, dass es sich lediglich um die Meinung von Juristen handelt, wobei verschiedene Juristen auch noch divergierende Meinungen vertreten können. Die Meinung von Juristen ist auch nicht per se ethisch-moralisch richtig. Dies gilt letztendlich für die Ansicht eines jeden Menschen, auch für die Ansicht eines Theologen. Auch er äußert seine persönliche Meinung, die darüber Aufschluss gibt, wie er bestimmte Teile der Bibel theologisch interpretiert. Von anderen Personen, auch von anderen Theologen, kann dies anders interpretiert werden. Cesetze und die Rechtsprechung sind nicht identisch mit dem moralischen Recht. $\mathrm{Zu}$ unterscheiden sind die ethisch-moralische Perspektive, die juristische, die theologische, die medizinische etc.

Ein Problem wird bleiben, zu entscheiden, wann bei einer Person Autonomie vorhanden ist, wann sie urteilsfähig, wann fremdbestimmt ist. Eine einfache Antwort hierauf gibt es ebenfalls nicht. Jeder einzelne Fall muss sorgfältig bewertet werden. Für das generelle Verhalten kann es daher nur wenige Regeln geben. Falls man bei der Entscheidung unsicher ist, ist dies gut. Derartige Entscheidungen fallen letztendlich nicht $100 \mathrm{zu}$ o aus, sondern beispielsweise 6o zu 40. Die Entscheidung könnte ein Jahr später auch umgekehrt ausfallen.

Es empfiehlt sich, bei schwierigen Fragen ein Ethikkomitee einzuschalten und mit diesem über den Fall zu beraten (Maio 2012). In diesem oder einem ähnlichen Gremium sollten möglichst auch Vertreter unterschiedlicher Gruppen sitzen, beispielsweise Ärzte, Pflegepersonal und Notfallseelsorger. Für die Entscheidung stehen diverse Modelle zur Verfügung (Rabe 2012). Für eine Entscheidung sollten die Personen nicht nur beruflich kompetent sein, sondern auch moralisch-ethisch geschult. Qualifizierungsmöglichkeiten finden sich z.B. unter http://www.aem-online.de/ oder http://www.ethikkomitee.de/zertifizierung/zertifizierung/index.html.

Vergessen werden sollte nicht, dass bei der Entscheidung das eigene Wertesystem und die persönliche Lebenseinstellung eine zentrale Rolle spielen. Häufig wird das eigene Wertesystem nicht hinterfragt, sondern generalisiert, ohne dass realisiert wird, dass es sich um eine unzulässige Generalisierung handelt.

\section{Zusammengefasst:}

- Entscheiden Sie bei jedem Patienten individuell.

- Sprechen Sie vor einer Entscheidung mit anderen Personen über Ihre Entscheidung.

- Rufen Sie bei schwierigen Entscheidungen ein Ethikkomitee an. 
- Versuchen Sie nicht, durch endloses Nachdenken die objektiv richtige Lösung zu finden - es gibt sie nicht.

- Machen Sie sich klar, dass Ihre Entscheidung letztendlich von Ihrem Weltbild abhängt.

- Falls für eine Diskussion keine Zeit verbleibt, entscheidet die ranghöchste Person.

- Sprechen Sie nach einer Entscheidung mit anderen Personen.

- Sprechen Sie in einer Supervisionsgruppe über Ihre Entscheidung und über ihr Empfinden in derartigen Situationen.

\section{Literatur}

Dilling H, Mombour W, Schmidt M, Schulte-Markwort E, World Health Organization WHO Press Mr lan Coltart (Hrsg.) (2011) Internationale Klassifikation psychischer Störungen. ICD 10. Bern, Hans Huber

Falkai P, Wittchen H-U (2015) (Hrsg.) Diagnostisches und Statistisches Manual Psychischer Störungen DM-5. Göttingen, Hogrefe

Fenner D (2008) Suizid - Krankheitssymptom oder Signatur der Freiheit? München, Verlag Karl Albert

Hick C, Bengel I, Mohr M, Reiter-Theil S (2000) Ethik in der präklinischen Notfallversorgung. Nottuln, Verlag- und Vertriebsgesellschaft des DRK

Lasogga F (2011) Überbringen einer Todesnachricht. In: Lasogga F, Gasch B (Hrsg.) Notfallpsychologie - Ein Lehrbuch für die Praxis. 2. Auflage. Berlin, Springer, S. 347-356

Lasogga F, Karutz H (2012) Hilfen für Helfer. 2. Auflage. Edewecht, Stumpf \& Kossendey

Maio G (2012) Ethik in der Medizin - eine praxisbezogene Einführung. In: Salomon F (Hrsg.) Praxisbuch Ethik in der Intensivmedizin. 2. Auflage. Berlin, Medizinische Wissenschaftliche Verlagsgesellschaft, S. 1-18

Rabe M (2012) Ethische Reflexion und Entscheidungsfindung in der intensivmedizinischen Praxis. In: Salomon F (Hrsg.) Praxisbuch Ethik in der Intensivmedizin. 2. Auflage. Berlin, Medizinische Wissenschaftliche Verlagsgesellschaft, S. 29-40

Wikipedia: Terri Schiavo. Zugriff am 26.3.2015

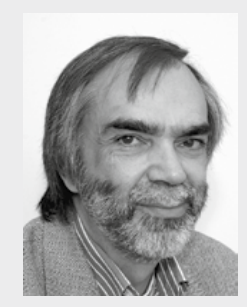

\section{Prof. Dr. Frank Lasogga}

Herr Professor Lasogga studierte Psychologie an der Universität Hamburg. Seit 1975 lehrt und forscht er an der Universität Dortmund. Hauptarbeitsgebiet: Klinische Psychologie mit dem Schwerpunkt Notfallpsychologie (Psychische Erste Hilfe, Psychosoziale Notfallhilfe; Hilfen für Helfer etc.). Seit 1988 hat er zahlreiche Bücher und Artikel zur Notfallpsychologie verfasst. 